\title{
Goodpasture's Syndrome With Ambiguous Serology: A Case Report
}

\author{
Joanna Szczykowska a, c, Szymon Brzosko a, Maja Rakowskab, \\ Beata Naumnik $^{\mathrm{a}}$
}

\begin{abstract}
Pulmonary-renal syndrome is a rare clinical syndrome defined by a combination of diffuse alveolar hemorrhage and rapidly progressive crescentic glomerulonephritis. It is not a single entity, but is caused by a wide variety of diseases including Goodpasture's syndrome associated with autoantibodies against glomerular and alveolar basement membrane (anti-glomerular basement membrane (anti-GBM)). Anti-GBM disease is an autoimmune disorder, estimated to occur in less than one case per million population. A 59-year-old previously healthy man presented with acute kidney injury (AKI, stage 3, KDIGO) with signs of nephritic syndrome, progressive oliguria, dyspnea with mild hemoptysis, cruses edema and elevated blood pressure. Serologic results were all negative except very low titer of anti-proteinase 3 antibodies. Chest radiograph revealed diffuse pulmonary infiltrations. Renal biopsy demonstrated necrotizing crescentic glomerulonephritis with linear pattern for immunoglobulin $\mathrm{G}$ along the entire GBM in immunofluorescent staining. Based on whole clinical picture, the diagnosis of Goodpasture's syndrome was done and the intensive treatment, including plasmapheresis, cyclophosphamide and prednisone, along with hemodialysis was provided. Improvement of patient's general condition was achieved. Despite recovery of diuresis, attempt to discontinue hemodialysis was found unsuccessful. The case proves the importance of diagnostic vigilance in patients presenting with AKI. Although supportive, in many cases, serology tests can be negative or ambiguous and should not prevent profound diagnostic process including kidney biopsy. Late diagnosis in the course of anti-GBM disease, in spite of proper treatment, correlates with less favorable kidney outcomes.
\end{abstract}

Keywords: Anti-GBM; Goodpasture syndrome; RPGN; Crescentic glomerulonephritis; Pulmonary-renal syndrome

Manuscript accepted for publication February 15, 2017

${ }^{a} 1$ st Department of Nephrology and Transplantation With Dialysis Unit, Medical University of Bialystok, Bialystok, Poland

'Students' Scientific Society, Medical University of Bialystok, Bialystok, Poland

${ }^{\mathrm{c} C o r r e s p o n d i n g ~ A u t h o r: ~ J o a n n a ~ S z c z y k o w s k a, ~ 1 s t ~ D e p a r t m e n t ~ o f ~ N e p h r o l-~}$ ogy and Transplantation With Dialysis Unit, Medical University of Bialystok, Zurawia 14, 15-540 Bialystok, Poland. Email: a.szczykowska@wp.pl

doi: https://doi.org/10.14740/wjnu301w

\section{Introduction}

Pulmonary-renal syndrome is a severe clinical syndrome, defined by a combination of diffuse alveolar hemorrhage and rapidly progressive crescentic glomerulonephritis. It is not a single entity, but is caused by a wide variety of diseases. Numerous studies have found that the most common cause is antineutrophil cytoplasmic antibodies (ANCA)-positive vasculitis followed by another autoimmune disorder anti-glomerular basement membrane (anti-GBM) antibody disease or Goodpasture's syndrome (GS) [1-3]. The incidence of anti-GBM disease is estimated to be $0.5-1.8$ cases per million population [4]. It is responsible for $1-5 \%$ of all types of glomerulonephritis and for $10-20 \%$ of crescentic glomerulonephritis [5]. This medical condition is associated with presence of circulating autoantibodies against $\alpha 3$ chain of type IV collagen localized in glomerular and alveolar basement membrane. Although rare, a few cases with absence of circulating anti-GBM antibodies have been described $[6,7]$.

This case review focuses on a classic clinical presentation of anti-GBM disease with supporting histologic and radiologic evidence but with ambiguous serological evidence.

\section{Case Report}

A 59-year-old previously healthy man, a long-standing cigarette smoker, presented to a local community hospital emergency department with acute kidney injury (AKI) with progressive oliguria, dyspnea, cruses edema and elevated blood pressure. Two weeks before hospitalization, he had lumbar pain, polyuria, hematuria and proteinuria and was unsuccessfully treated with ciprofloxacin. There was no another previous history of renal or pulmonary disease. No family disease was known and he had also no other relevant findings in his past medical history. Upon admission, his chief complaint was malaise and weakness. Laboratory data revealed elevated levels of serum creatinine of $10.3 \mathrm{mg} / \mathrm{dL}$, fulfilling the requirements of third stage of AKI according to KDIGO criteria, blood urea nitrogen level of $78.5 \mathrm{mg} / \mathrm{dL}$, normocytic normochromic anemia with hemoglobin level of $8.8 \mathrm{~g} / \mathrm{dL}$ and slightly elevated C-reactive protein level of $22 \mathrm{mg} / \mathrm{L}$. Urinalysis showed active urine sediment with numerous dysmorphic red blood cells and non-nephrotic range proteinuria. Chest radiograph revealed 


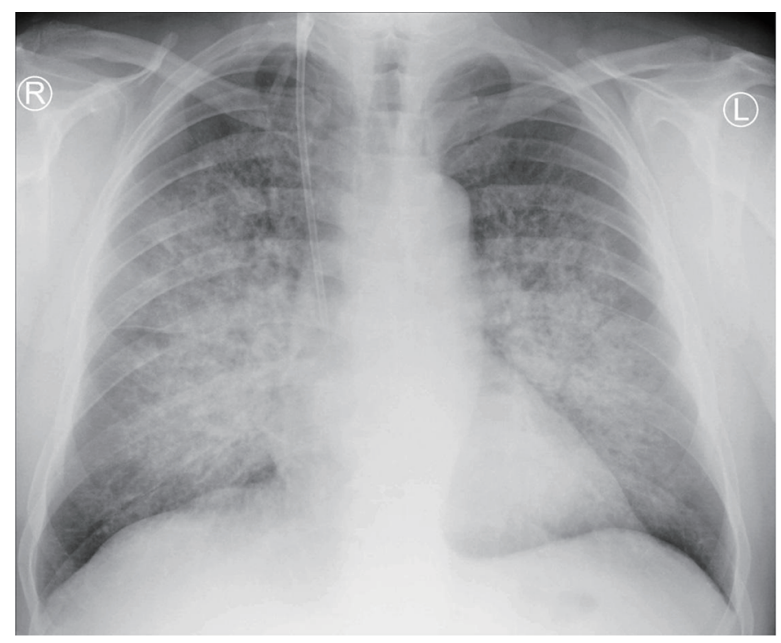

Figure 1. Chest X-ray revealing bilateral diffuse alveolar infiltration spreading from hilums.

diffuse pulmonary infiltration (Fig. 1). His oxygen saturation was $95 \%$ on $21 \%$ oxygen with no signs of carbon dioxide retention.

The patient was transferred to our institution for further workup of pulmonary-renal syndrome. Serologic tests for ANA, pANCA and anti-GBM antibodies were all negative except anti-proteinase 3 antibody (cANCA) at very low titers. Ultrasonography examination revealed kidney of normal size with no anatomic abnormalities, so the procedure of kidney biopsy was performed. Necrotizing crescentic rapidly progressive glomerulonephritis (RPGN) with linear pattern for immunoglobulin $\mathrm{G}$ along the entire GBM in immunofluorescent staining was identified. In three of six glomeruli, large cellular crescent and fibrinoid necrosis of capillaries were described (Figs. 2 and 3). The patient's respiratory status started to deteriorate and the episodes of mild hemoptysis appeared. Chest computed tomography (CT) revealed bilateral, coalescent airspace opacities and extensive ground glass appearance (Fig. 4).

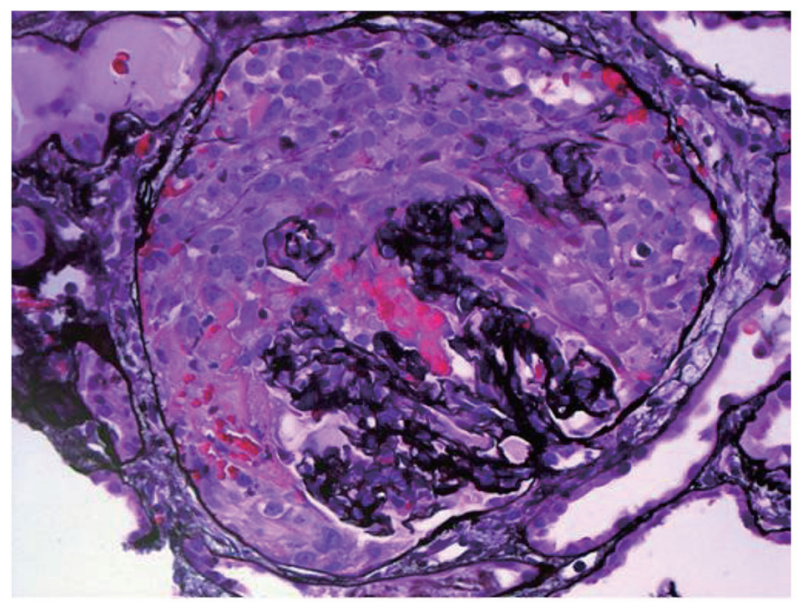

Figure 2. Light microscopy, hematoxylin and eosin stain showing large cellular crescent and fibrinoid necrosis of capillaries.

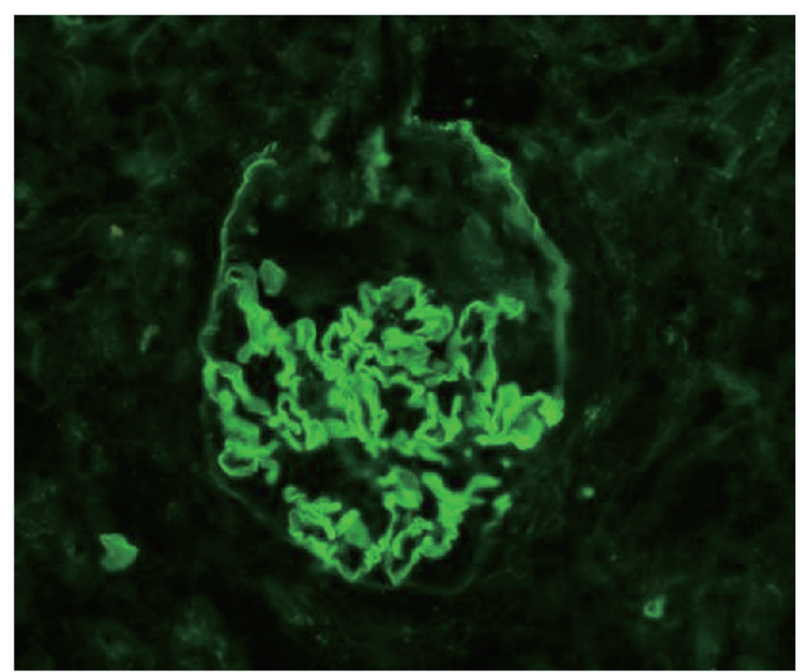

Figure 3. Immunofluorescentic staining. Linear pattern of immunoglobulin $\mathrm{G}$ along the entire glomerular basement membrane, typical for anti-GBM disease.

Based on whole clinical picture, the diagnosis of GS, despite lack of circulating anti-GBM antibodies, was made and the intensive treatment was provided. It included 14 plasmapheresis to remove potential circulating antibodies and other mediators of inflammation, combined with oral cyclophosphamide and methyloprednisolone for first 3 days intravenously, followed by daily oral prednisone. Doses were determined according to KDIGO 2012 guidelines. Hemodialysis three times per week was also continued. Improvement of patient's general condition was achieved. Unfortunately, despite recovery of diuresis (even 2,000 mL/day), attempt to discontinue renal replacement therapy was found unsuccessful, as the continuous rise of creatinine concentration was observed.

\section{Discussion}

GS typically occurs in the third and seventh decade of life. Symptoms may either start slowly, gradually affecting kidneys and lungs, or they may progress rapidly, and become severe in a few days [8]. There are essential variations in the clinical manifestations of patients with anti-GBM disease, with 60$80 \%$ having clinically apparent pulmonary and renal involvement, $20-40 \%$ having renal disease only, and less than $10 \%$ having only pulmonary manifestation [9]. Our case had a classic bimodal presentation with an initial more expressed renal degradation phase, followed by pulmonary involvement.

RPGN and diffuse alveolar hemorrhage represent a medical emergency, so an early diagnosis and appropriate treatment should have been provided. The prognosis of untreated acute glomerulonephritis due to GS is extremely poor. In one review of 32 patients suffering from anti-GBM disease, 29 (which is $90 \%$ ) progressed to end-stage renal disease, most of them in less than 6 months [10]. The use of aggressive therapy with plasma exchange in association with immunosuppressive agents has dramatically improved outcome [11]. However, 


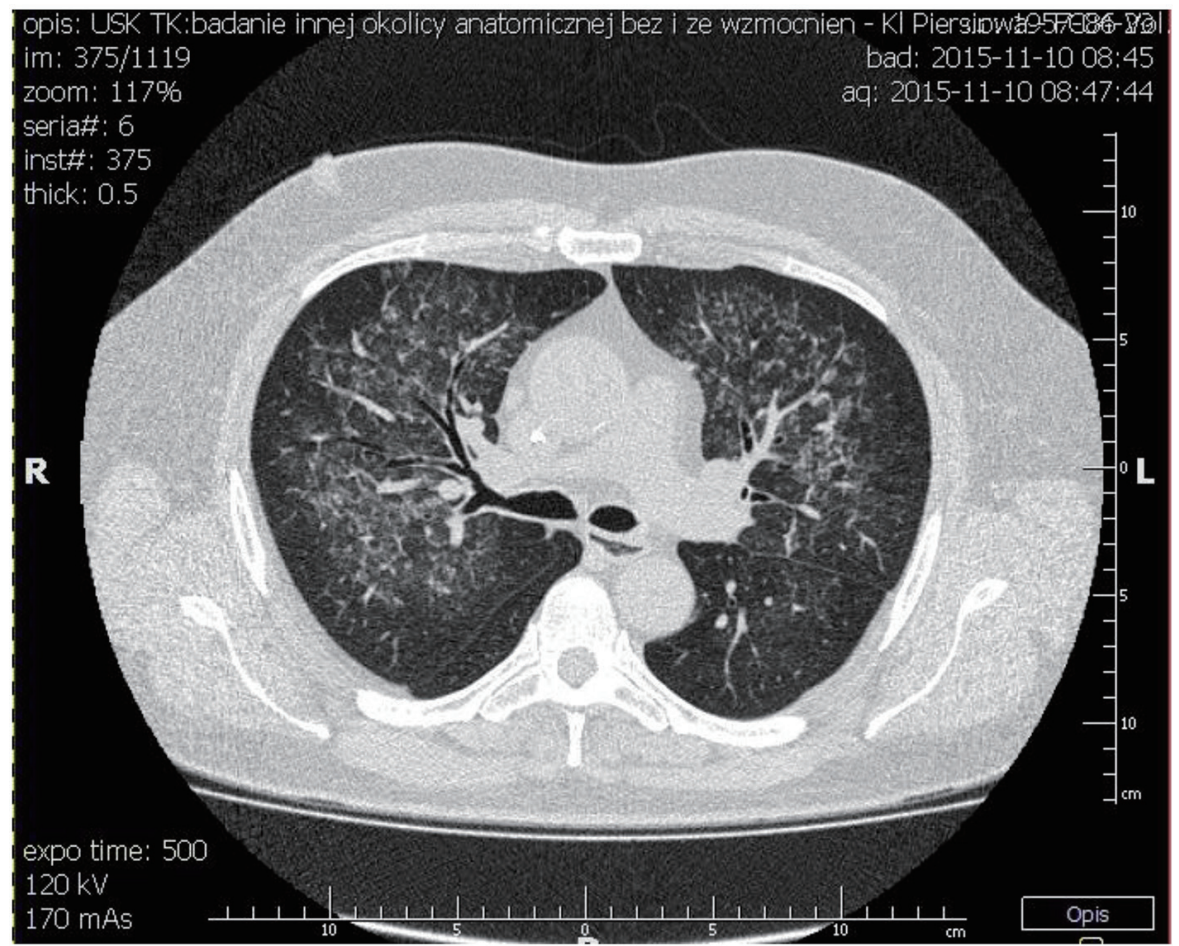

Figure 4. Chest CT showing bilateral, coalescent airspace opacities and extensive ground glass appearance spreading from bronchi.

renal survival still correlates closely with the degree of renal failure at presentation [12]. Therefore, an early diagnosis is essential for patient survival and recovery of renal function.

The diagnosis of pulmonary-renal syndrome caused by anti-GBM disease requires demonstration of anti-GBM antibodies either in the serum or the kidney. In our case test for circulating anti-GBM antibodies was negative. It might have been false negative result, because it was performed using indirect immunofluorescence on primate kidney and lung tissue, which is not quantitative and sensitive enough and can give false negative results even in 10-40\% [13]. A more common approach is detection of anti-GBM antibodies in serum using an enzyme-linked immunosorbent assay (ELISA), but it is unfortunately not routinely performed in our laboratory. This assay has reported sensitivity of over $95 \%$ and specificity of $91-100 \%$ [10]. Nonetheless, there are several cases in which no circulating anti-GBM antibodies were detectable by well-established ELISA or Western blotting techniques. One of them describes three cases where diagnosis of GS was confirmed by renal biopsy. In addition, an alternative method of antibody detection using highly sensitive biosensor system confirmed that circulating antibodies were present in sera from both patients tested [6]. Because this technique is not widely available for the detection of anti-GBM antibodies, the diagnosis of anti-GBM disease should be considered in correct clinical context, even despite negative serological test results and always be confirmed by renal biopsy.

Not only anti-GBM antibodies were equivocal in our case, but also was test for cANCA positive. It is not so rare phenomenon. One-third of patients with GS, during the natural course of illness, have circulating antineutrophilic cytoplasmic antibodies in addition to anti-GBM antibodies [14]. However, numerous studies have shown that ANCA positive patients with anti-GBM disease usually have ANCA directed against myeloperoxidase (pANCA) rather than proteinase-3 (cANCA) [14, 15]. In most cases, the ANCA antibodies may be detectable months or years before the production of anti-GBM antibodies and clinical onset of the disease [16]. There is a hypothesis that renal involvement in ANCA vasculitis leads to the exposure of antigens from the basement membrane and the formation of specific antibodies.

The treatment of choice in GS is plasmapheresis in conjunction with oral prednisone and cyclophosphamide. This aggressive regimen has significantly improved prognosis. Regrettably, as in our case, recovery of kidney function is rare if dialysis is required at initiation of treatment [12]. Moreover, the best therapy response is achieved when the percent of crescent on initial kidney biopsy is lower than 50 and pretherapy serum creatinine concentration is below $4.0 \mathrm{mg} / \mathrm{dL}$ [5]. This was confirmed also in our case.

\section{Conclusions}

GS is a rare autoimmune disorder with many types of presentation but it always has to be considered in patients presenting with rapidly progressive AKI, despite the absence of antiGBM antibodies. Seronegative pulmonary-renal syndrome is a challenging entity to the clinician, since early diagnosis may be missed leading to delayed appropriate treatment. Negative 
or ambiguous serology test should not prevent profound diagnostic process. Each case of AKI of unknown etiology requires renal biopsy to identify the underlying cause and to help direct therapy. Late diagnosis, even in spite of right treatment, correlates with less favorable kidney outcomes. The presence of cANCA adds complexity to the diagnostic approach in an already rare and complex disease.

\section{Conflicts of Interest}

The authors declare no conflicts of interest.

\section{References}

1. Niles JL, Bottinger EP, Saurina GR, Kelly KJ, Pan G, Collins AB, McCluskey RT. The syndrome of lung hemorrhage and nephritis is usually an ANCA-associated condition. Arch Intern Med. 1996;156(4):440-445.

2. Boyce NW, Holdsworth SR. Pulmonary manifestations of the clinical syndrome of acute glomerulonephritis and lung hemorrhage. Am J Kidney Dis. 1986;8(1):31-36.

3. Gallagher H, Kwan JT, Jayne DR. Pulmonary renal syndrome: a 4-year, single-center experience. Am J Kidney Dis. 2002;39(1):42-47.

4. Kluth DC, Rees AJ. Anti-glomerular basement membrane disease. J Am Soc Nephrol. 1999;10(11):2446-2453.

5. Tang W, McDonald SP, Hawley CM, Badve SV, Boudville NC, Brown FG, Clayton PA, et al. Anti-glomerular basement membrane antibody disease is an uncommon cause of end-stage renal disease. Kidney Int. 2013;83(3):503510.

6. Salama AD, Dougan T, Levy JB, Cook HT, Morgan SH, Naudeer S, Maidment G, et al. Goodpasture's disease in the absence of circulating anti-glomerular basement membrane antibodies as detected by standard techniques. Am J Kidney Dis. 2002;39(6):1162-1167.
7. Fernandes R, Freitas S, Cunha P, Alves G, Cotter J. Goodpasture's syndrome with absence of circulating anti-glomerular basement membrane antibodies: a case report. J Med Case Rep. 2016;10:205.

8. Dammacco F, Battaglia S, Gesualdo L, Racanelli V. Goodpasture's disease: a report of ten cases and a review of the literature. Autoimmun Rev. 2013;12(11):11011108.

9. Hellmark T, Segelmark M. Diagnosis and classification of Goodpasture's disease (anti-GBM). J Autoimmun. 2014;48-49:108-112.

10. Wilson CB, Dixon FJ. Anti-glomerular basement membrane antibody-induced glomerulonephritis. Kidney Int. 1973;3(2):74-89.

11. Lockwood CM, Rees AJ, Pearson TA, Evans DJ, Peters $\mathrm{DK}$, Wilson CB. Immunosuppression and plasma-exchange in the treatment of Goodpasture's syndrome. Lancet. 1976;1(7962):711-715.

12. Levy JB, Turner AN, Rees AJ, Pusey CD. Long-term outcome of anti-glomerular basement membrane antibody disease treated with plasma exchange and immunosuppression. Ann Intern Med. 2001;134(11):1033-1042.

13. Wilson CB, Dixon FJ. Diagnosis of immunopathologic renal disease. Kidney Int. 1974;5(6):389-401.

14. Weber MF, Andrassy K, Pullig O, Koderisch J, Netzer K. Antineutrophil-cytoplasmic antibodies and antiglomerular basement membrane antibodies in Goodpasture's syndrome and in Wegener's granulomatosis. J Am Soc Nephrol. 1992;2(7):1227-1234.

15. Hellmark T, Niles JL, Collins AB, McCluskey RT, Brunmark C. Comparison of anti-GBM antibodies in sera with or without ANCA. J Am Soc Nephrol. 1997;8(3):376385.

16. Olson SW, Arbogast CB, Baker TP, Owshalimpur D, Oliver DK, Abbott KC, Yuan CM. Asymptomatic autoantibodies associate with future anti-glomerular basement membrane disease. J Am Soc Nephrol. 2011;22(10):19461952. 